\title{
半乳糖胺修饰阳离子型刷形嵌段共聚物的合成与表征
}

\author{
郝莹张洋何金林尚修娟张明祖倪沛红* \\ (苏州大学材料与化学化工学部 江苏省先进功能高分子材料设计及应用重点实验室 苏州 215123)
}

\begin{abstract}
摘要 利用两步原子转移自由基聚合(ATRP)和聚合物后修饰反应，制备半乳糖胺修饰的阳离子型刷形嵌段共聚物 P(PEGMEMA-co-PEGMA-Gal)- $b$-PDMAEMA．首先，通过 ATRP 法引发单体聚乙二醇单甲醚甲基丙烯酸酯(PEGMEMA) 和聚乙二醇甲基丙烯酸酯(PEGMA)聚合, 得到末端含氯基的无规共聚物 P(PEGMEMA-co-PEGMA); 再利用其作为大 分子引发剂, 引发水溶性单体甲基丙烯酸-2-( $N, N$-二甲氨基)乙酯(DMAEMA)进行 ATRP 反应，获得三组分两嵌段刷形 共聚物 P(PEGMEMA-co-PEGMA)- $b$-PDMAEMA; 最后, 利用 PEGMA 结构单元中的侧链羟基, 经聚合物反应, 键合具 有肝靶向功能的半乳糖分子 (Gal), 成功获得 P(PEGMEMA-co-PEGMA-Gal)- $b$-PDMAEMA. 其中, PDMAEMA 侧基的二 甲氨基 $\left[-\mathrm{N}\left(\mathrm{CH}_{3}\right)_{2}\right]$ 在中性和弱酸性介质中可发生质子化, 形成聚阳离子链段, 能够缩合 DNA, 用作基因载体. 通过核 磁共振氢谱 $\left({ }^{1} \mathrm{H}\right.$ NMR)、红外光谱(FT-IR)和凝胶渗透色谱(GPC)，对聚合物的化学结构、分子量及分子量分布进行表征. 利用凝胶阻滞电泳研究阳离子型嵌段共聚物与 DNA 的结合能力、利用动态激光光散射仪(DLS)测试聚阳离子/DNA 复 合物的表面 zeta 电位值、粒径及粒径分布. 通过细胞毒性试验(MTT 法)表征载体的生物相容性, 并分别研究复合物对 宫颈癌(HeLa)细胞和肝癌(HepG2)细胞的转染能力. 实验结果表明, 这种半乳糖胺修饰的阳离子型刷形共聚物具有较低 的细胞毒性，在肝靶向基因输送中具有潜在的应用.
\end{abstract}

关键词 半乳糖胺; 刷形共聚物; 原子转移自由基聚合; 基因载体

\section{Synthesis and Characterization of PEGylated Brush-type Polycation Modified with Galactosamine}

\author{
Hao, Ying Zhang, Yang He, Jinlin Shang, Xiujuan Zhang, Mingzu Ni, Peihong* \\ (College of Chemistry, Chemical Engineering and Materials Science, Jiangsu Key Laboratory of Advanced Functional \\ Polymer Design and Application, Soochow University, Suzhou 215123)
}

\begin{abstract}
Gene therapy has been generally regarded as a promising treatment for numerous hard curable diseases, such as cancer, genetic and infectious diseases. Seeking a safe and efficient vector plays the most challenging role in gene therapy. In the past decades, non-viral polycation has attracted much more attentions as a promising gene vector due to their good biocompatibility, high condensation ability, as well as easily-adjustable and controlled structures. In the present work, a series of galactosamine $(\mathrm{Gal})$-conjugated brush-type cationic copolymer P(PEGMEMA-co-PEGMA -Gal $_{\text {- }}$ - $b$-PDMAEMA were prepared via a combination of two-step atom transfer radical polymerization (ATRP) technique and polymer reaction. Firstly, random copolymerization of poly(ethylene glycol)methyl ether methacrylate (PEGMEMA) and poly(ethylene glycol) methacrylate (PEGMA) was carried out to yield the copolymer P(PEGMEMA-co-PEGMA), which was further used as a macroinitiator to polymerize 2-( $N, N$-dimethylamino)ethyl methacrylate (DMAEMA), producing the cationic copolymer P(PEGMEMA-co-PEGMA)- $b$-PDMAEMA. Finally, the brush-type copolymer P(PEGMEMA-co-PEGMA-Gal)- $b$ PDMAEMA was obtained by the modification of pendent hydroxyl groups with galactosamine using $N, N^{\prime}$-carbonyldiimidazole (CDI) as the coupling agent. PDMAEMA is a well-known pH-sensitive polycation and has been widely applied for non-viral gene delivery, while the brush-type hydrophilic chains provide the carrier with favorable biocompatibility, prolonged blood circulation time, and reduced non-specific adsorption of proteins. PDMAEMA block would be partially protonated to afford some hydrophobic domains and positive charges at neutral condition, which can bind with DNA via electrostatic interaction to form polycation/DNA polyplex. The Gal moiety on the surface made the polyplex easily recognized by asialoglycoprotein receptors (ASGPRs) over-expressing hepatoma cells and internalized via a receptor-mediated endocytosis process. The chemical structures, molecular weights and molecular weight distributions of P(PEGMEMA-co-PEGMA-Gal)- $b$-PDMAEMA copolymer were characterized by ${ }^{1} \mathrm{H}$ NMR, FT-IR, and GPC measurements. The DNA binding capacity of this copolymer was investigated by agarose gel electrophoresis and zeta potential measurements, which indicated that DNA migration could be completely retarded when the N/P ratio was higher than 1.5. The DLS
\end{abstract}

*E-mail: phni@suda.edu.cn; Tel.: 0512-65882047

Received March 15, 2014; published April 17, 2014.

Projects supported by the National Natural Science Foundation of China (Nos. 21374066 and 21074078), the Natural Science Foundation of Jiangsu Province for Rolling Support (No. BK2011045), the Priority Academic Program Development (PAPD) of Jiangsu Higher Education Institutions.

受国家自然科学基金(Nos. 21374066, 21074078)、江苏省基础研究计划(自然科学基金)——滚动资助项目(No. BK2011045)、江苏高校优势学科建设项 目资助. 
analysis demonstrated that the polyplex held an average particle size of about $106 \mathrm{~nm}$. In addition, in vitro cytotoxicity (MTT assay) and transfection assays were also examined, the results showed that the copolymer possessed relatively lower cytotoxicity than branched PEI $(25 \mathrm{kDa})$, and the transfection of polyplex into HepG2 cells was more efficient than that of HeLa cells studied by live cell imaging system owing to specific ligand-receptor interactions between Gal and ASGPRs on the surface of HepG2 cells. This study presents a facile strategy for the preparation of biocompatible polymeric micelles that will act as a promising platform for hepatoma-targeting delivery of genes.

Keywords galactosamine; brush-type copolymer; ATRP; gene delivery

\section{1 引言}

随着基因治疗的不断深入研究, 其在治疗遗传病、 肿瘤等多种疾病中取得了突破性进展. 目前, 用于基因 治疗的载体主要有病毒载体和非病毒载体. 非病毒载体 由于其具有低免疫反应、结构可控和易于组装等优点而 引起广泛关注 ${ }^{[1 \sim 4]}$. 作为一类常用的非病毒基因载体, 阳离子型聚合物具有较高的转运能力和转染效率, 但同 时也存在一些问题, 例如: 稳定性较差、细胞毒性较高, 易与人体血液中的带负电荷组分结合而导致非特异性 清除等. 为了解决这些缺点, 研究者一般是通过对阳离 子型聚合物进行功能化修饰, 以降低载体的细胞毒 性 ${ }^{[5 \sim 10]}$ 、并提高其转染效率 ${ }^{[11 ~ 14]}$. 研究发现, 通过引入 一些亲水性聚合物链段 ${ }^{[15]}$, 如聚乙二醇(PEG)和聚甲基 丙烯酸着丙酯(PHPMA)等, 可以屏蔽载体表面的正电 荷. 而具有高密度侧链的刷形聚合物能够改变聚阳离子 主链周围的微环境, 尤其是基于 PEG 的刷形聚合物, 可 以屏蔽正电荷、降低载体毒性、延长载体在体内的循环 时间, 并且具有抗蛋白非特异吸附性能 ${ }^{[16 ~ 18]}$.

另一方面, 由于体内调理素作用以及肝脏微血管的 非连续内皮连接, 通过静脉注射的纳米粒子载体可在短 时间内抵达肝脏富集. 然而, 肝脏滤过是纳米粒子遇到 的主要屏障, 大多数纳米粒子会迅速被 Kupffer 细胞从 体内清除, 并不会通过内吞作用进入肝细胞. 因此, 在 纳米粒子表面修饰具有肝靶向功能的配体分子, 可以增 强其对肝细胞的靶向能力 ${ }^{[19-22]}$. 半乳糖分子是其中一 种重要配体, 它能够与哺乳类动物肝细胞表面过度表达 的外源凝集素发生特异性作用, 从而增强基因或药物的 输送.

本文设计合成了半乳糖胺修饰的阳离子型刷形嵌 段共聚物 P(PEGMEMA-co-PEGMA-Gal)- $b$-PDMAEMA.
如图 1 所示, 聚合物中 PDMAEMA 链段在中性条件下 (pH 7.4)发生部分质子化带正电荷, 可以与带负电荷的 DNA 通过静电作用相结合, 形成外围含具有抗蛋白吸 附能力的亲水性刷形 PEG 链段的复合物; 同时, 复合物 表面带有靶向分子半乳糖，在肝靶向基因治疗中具有潜 在的应用前景.

\section{2 结果与讨论}

\section{1 结构表征}

2.1.1 P(PEGMEMA-co-PEGMA)及 P(PEGMEMA-coPEGMA)- $b$-PDMAEMA 的结构表征

图 2 为本文涉及的几种共聚物的合成路线. 首先利 用 ${ }^{1} \mathrm{H}$ NMR 验证无规共聚物 P(PEGMEMA-co-PEGMA) 的结构 ${ }^{[23,24]}$, 结果如图 3(A)所示. ${ }^{1} \mathrm{H}$ NMR(DMSO- $d_{6}$ ) 谱 图中的代表性化学位移如下: $\delta \sim 4.0$ [f 峰, P(PEGMEMA-co-PEGMA) 侧链中- $\mathrm{COOCH}_{2}$ 一上的 H]; $\delta \sim 3.0$ [h 峰, P(PEGMEMA-co-PEGMA) 侧链中 $-\mathrm{O}-\mathrm{CH}_{3}$ 上的 $\mathrm{H}$ ]; $\delta 4.5 \sim 4.6$ [j 峰, PEGMA 侧链中 $-\mathrm{CH}_{2} \mathrm{OH}$ 上的 $\left.\mathrm{H}\right]$. 从而可以证明成功制得末端含氯基 的无规共聚物 P(PEGMEMA-co-PEGMA).

利用 P(PEGMEMA-co-PEGMA)作为大分子 ATRP 引发剂, 引发单体 DMAEMA 进行聚合, 获得阳离子型 嵌段共聚物 P(PEGMEMA- $c o$-PEGMA)- $b$-PDMAEMA. 图 3(B) 为嵌段共聚物的 ${ }^{1} \mathrm{H}$ NMR 谱图, 从图中可以找到 相对应各个质子的归属, 初步证明已经成功制得嵌段共 聚物. ${ }^{1} \mathrm{H}$ NMR $\left(\mathrm{DMSO}-d_{6}\right.$ ) 谱图中的代表性化学位移: $\delta \sim 3.5$ [j 峰, PDMAEMA 链段中一 $\mathrm{CH}_{2} \mathrm{~N}\left(\mathrm{CH}_{3}\right)_{2}$ 上的 $\mathrm{H}] ; \delta \sim 4.0$ [f 峰, PDMAEMA 链段侧链中 $-\mathrm{COOCH}_{2}$-上的 $\mathrm{H}$ ]; $\delta \sim 2.2$ [ $\mathrm{k}^{\prime}$ 峰, PDMAEMA 侧链

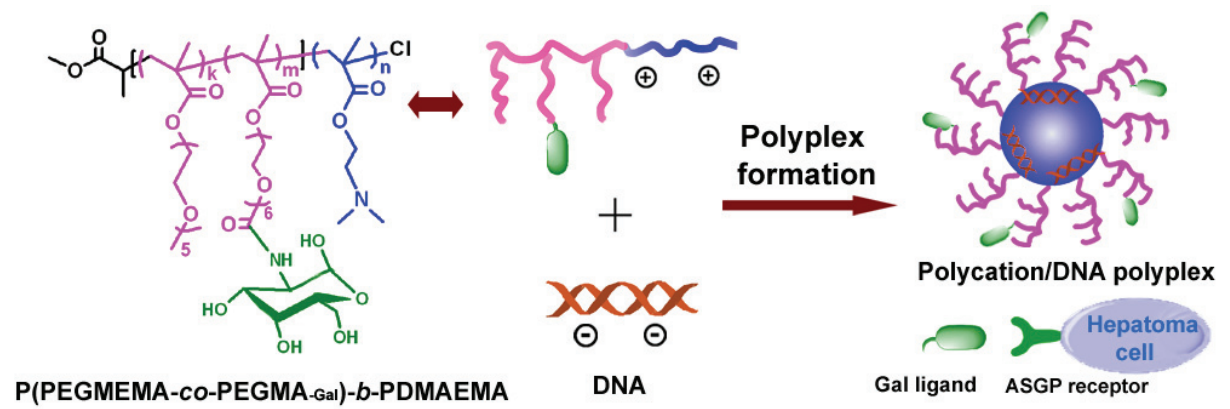

图 1 半乳糖胺修饰的阳离子型刷形嵌段共聚物 P(PEGMEMA-co-PEGMA-Gal)- $b$-PDMAEMA 与 DNA 形成复合物的组装示意图 Figure 1 Schematic depiction of the formation of P(PEGMEMA-co-PEGMA-Gal)- $b$-PDMAEMA/DNA polyplex 
中一 $\mathrm{N}\left(\mathrm{CH}_{3}\right)_{2}$ 上的 $\left.\mathrm{H}\right]$. 进一步通过 GPC 表征两种共聚物 P(PEGMEMA-co-PEGMA) 及 P(PEGMEMA-coPEGMA)- $b$-PDMAEMA 的分子量及分子量分布, 结果 如表 1 所示, 从嵌段共聚物分子量的增长可以验证成功 合成了目标产物.
根据图 3 中聚合物的特征化学位移峰面积，可以利 用下列公式计算得到各个嵌段的聚合度:

$$
\frac{A_{\mathrm{c}}}{3}=\frac{A_{\mathrm{h}}}{3 k} ; \quad \frac{A_{\mathrm{c}}}{3}=\frac{A_{\mathrm{j}}}{m} ; \quad \frac{A_{\mathrm{c}}}{3}=\frac{A_{\mathrm{k}^{\prime}}}{6 n}
$$

(1)<smiles>C=C(C)C(=O)OCCOC(=O)OCCN</smiles>

(2)

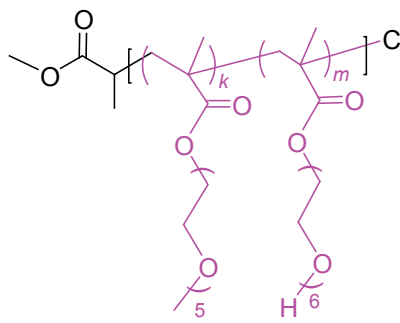<smiles>C=C(C)C(=O)OCCN(C)C</smiles><smiles>CCNOCC</smiles><smiles>CCCONC(=O)OCC</smiles>

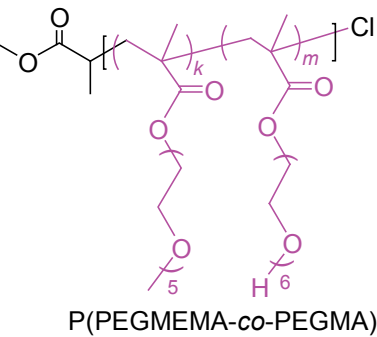

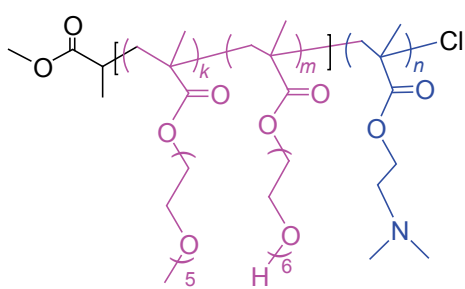

P(PEGMEMA-Co-PEGMA)-b-PDMAEMA
P(PEGMEMA-co-PEGMA_CDI)- $b$-PDMAEMA

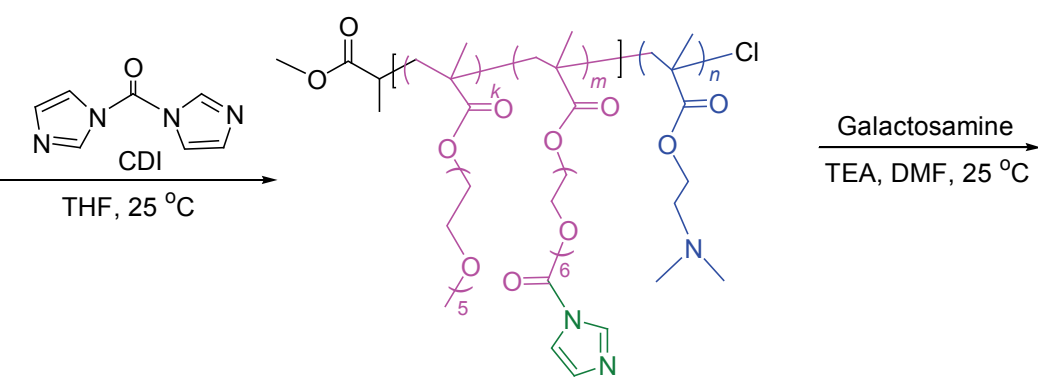

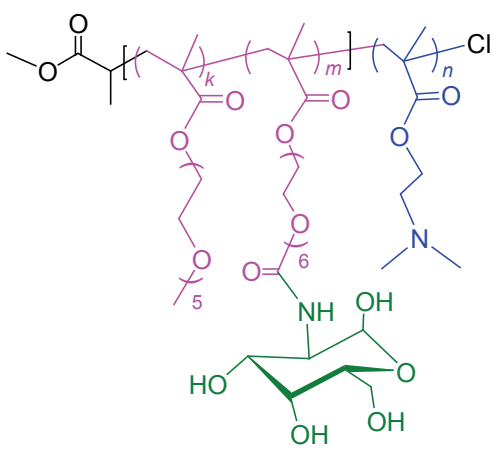

P(PEGMEMA-co-PEGMA_Gal)-b-PDMAEMA

图 2 半乳糖胺修饰的阳离子型刷形嵌段共聚物 P(PEGMEMA-co-PEGMA-Gal)- $b$-PDMAEMA 的合成路线

Figure 2 Synthesis route of P(PEGMEMA-co-PEGMA-Gal)-b-PDMAEMA copolymer
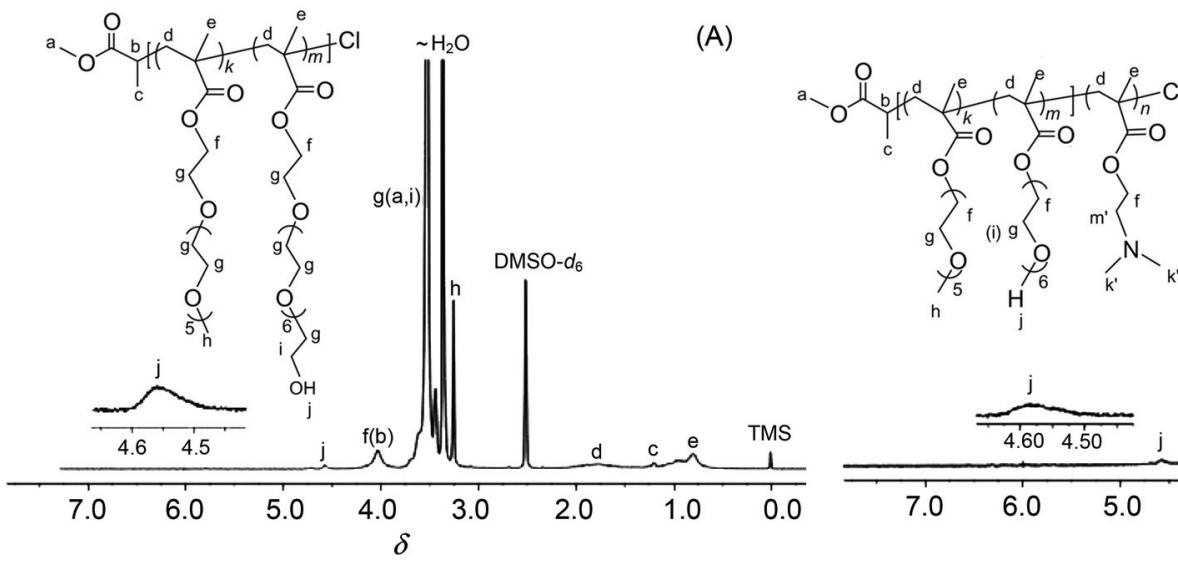

(B)

图 3 两种共聚物的 ${ }^{1} \mathrm{H}$ NMR 谱图: (A) P(PEGMEMA $\left.{ }_{16}-c o-\mathrm{PEGMA}_{7}\right)$ 和(B) P(PEGMEMA $\left.{ }_{16}-c o-\mathrm{PEGMA}_{7}\right)-b-\mathrm{PDMAEMA}_{53}$

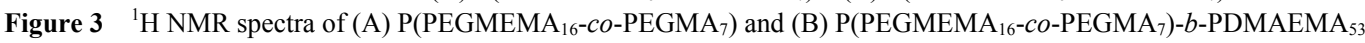


表 1 两种共聚物 P(PEGMEMA-co-PEGMA)和 P(PEGMEMA-co-PEGMA)- $b$-PDMAEMA 的化学组成、分子量及分子量分布

Table 1 Characterization data of the compositions, molecular weights, and molecular weight distributions of P(PEGMEMA-co-PEGMA) and P(PEGMEMA-co-PEGMA)- $b$-PDMAEMA copolymers

\begin{tabular}{|c|c|c|c|}
\hline Samples & $\bar{M}_{\mathrm{n}, \mathrm{GPC}} /\left(\mathrm{g} \cdot \mathrm{mol}^{-1}\right)$ & $\bar{M}_{\mathrm{w}, \mathrm{GPC}} /\left(\mathrm{g} \cdot \mathrm{mol}^{-1}\right)$ & PDI \\
\hline $\mathrm{P}\left(\mathrm{PEGMEMA}_{16}-\mathrm{Co}-\mathrm{PEGMA}_{6}\right)$ & 8670 & 11130 & 1.28 \\
\hline $\mathrm{P}\left(\mathrm{PEGMEMA}_{14}-\mathrm{co}-\mathrm{PEGMA}_{7}\right)$ & 8810 & 11430 & 1.30 \\
\hline $\mathrm{P}\left(\mathrm{PEGMEMA}_{16}-\mathrm{co}-\mathrm{PEGMA}_{7}\right)$ & 7650 & 9710 & 1.27 \\
\hline $\mathrm{P}\left(\mathrm{PEGMEMA}_{16}-\mathrm{co}-\mathrm{PEGMA}_{7}\right)$ & 7560 & 9510 & 1.26 \\
\hline $\mathrm{P}\left(\mathrm{PEGMEMA}_{21}-c o-\mathrm{PEGMA}_{3}\right)$ & 7460 & 9420 & 1.27 \\
\hline P(PEGMEMA $16-c o-$ PEGMA $\left._{6}\right)-b$-PDMAEMA 33 & 12390 & 20600 & 1.66 \\
\hline P(PEGMEMA $14-c o-$ PEGMA $\left._{7}\right)-b$-PDMAEMA 39 & 12960 & 21450 & 1.66 \\
\hline $\mathrm{P}_{\left(\mathrm{PEGMEMA}_{16}-c o-\mathrm{PEGMA}_{7}\right)-b-\mathrm{PDMAEMA}_{53}}$ & 15870 & 26980 & 1.70 \\
\hline
\end{tabular}

$\bar{M}_{n, \text { NMR, P(PEGMEMA-co-PEGMA })}=300 k+360 m+M_{\mathrm{MBP}}-80+35$

$\bar{M}_{n, \text { NMR, P(PEGMEMA-co-PEGMA)-b-PDMAEMA }}=\bar{M}_{n}$, NMR, P(PEGMEMA-co-PEGMA) $+157 n$

其中，字母 $\mathrm{A}$ 代表 ${ }^{1} \mathrm{H}$ NMR 谱图中特征峰的积分面积, 公式(1)和(2)分别对应图 3(A)和(B); 下标 $\mathrm{c}, \mathrm{h}, \mathrm{j}$ 和 $\mathrm{k}^{\prime}$ 分别 代表 ${ }^{1} \mathrm{H}$ NMR 谱图中所对应的化学位移， $k, m$ 和 $n$ 分别 代表三嵌段共聚物中 PPEGMEMA，PPEGMA 和 PDMAEMA 链段的聚合度, 300, 360 和 157 分别代表单 体 PEGMEMA, PEGMA 和 DMAEMA 的分子量, 80 和 35 分别为 $\mathrm{Br}$ 和 $\mathrm{Cl}$ 原子的相对原子量.

通过红外光谱(FT-IR)进一步验证聚合物的结构, 如图 4 所示, 其特征吸收峰的归属如下: 1250 1270 $\mathrm{cm}^{-1}\left(\mathrm{v}_{\mathrm{C}-\mathrm{O}-\mathrm{C}}\right) ; 1730 \mathrm{~cm}^{-1}\left(\mathrm{v}_{\mathrm{C}=\mathrm{O}}\right) ; 3450 \mathrm{~cm}^{-1}\left(\mathrm{v}_{-\mathrm{OH}}\right) ; 2845$ $\mathrm{cm}^{-1}\left(v_{\mathrm{C}-\mathrm{H}}\right) ; 2738 \mathrm{~cm}^{-1}\left[\mathrm{v}_{\mathrm{N}\left(\mathrm{CH}_{3}\right) 2}\right]$. 从图 4(A)可以观察到, 在 $3500 \mathrm{~cm}^{-1}$ 和 $1750 \mathrm{~cm}^{-1}$ 处分别为聚合物侧链上羟基 $(-\mathrm{OH})$ 以及羰基 $(\mathrm{C}=\mathrm{O})$ 的特征吸收峰; 图 4(B)中, 2730 $\mathrm{cm}^{-1}$ 处为刷形嵌段共聚物侧链上甲基 $\left(-\mathrm{CH}_{3}\right)$ 的特征吸 收峰.

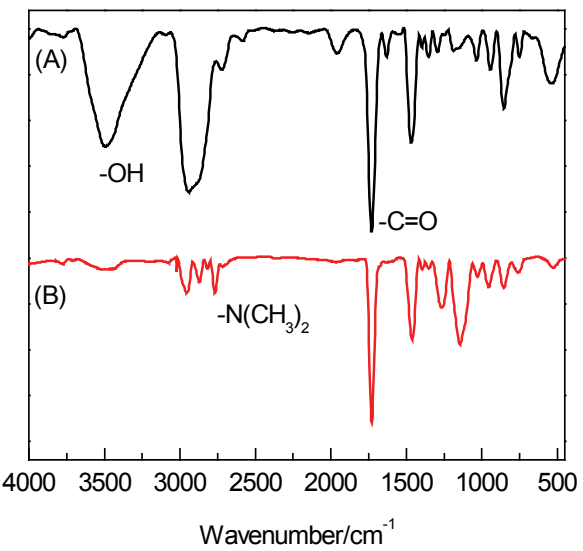

图 4 两种共聚物的红外光谱图: (A) P(PEGMEMA $\left.16-c o-\mathrm{PEGMA}_{7}\right)$; (B) P(PEGMEMA $16^{-}-$o- PEGMA $\left._{7}\right)-b$-PDMAEMA 53

Figure 4 FT-IR spectra of (A) P(PEGMEMA $16-$ - 0 - PEGMA $\left._{7}\right)$ and (B)

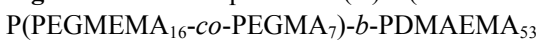

2.1.2 P(PEGMEMA-co-PEGMA-Gal)- $b$-PDMAEMA 的 结构表征

首先，利用 CDI 活化 PEGMA 结构单元侧链上的羟 基，接着与半乳糖胺反应，获得半乳糖胺修饰的阳离子 型刷形嵌段共聚物 P(PEGMEMA-co-PEGMA-Gal $)-b$ PDMAEMA. 从 ${ }^{1} \mathrm{H}$ NMR 谱图(图 5)中可以找到与各个 质子相对应的化学位移, 在 $\delta 8.2 \sim 8.6, \delta \sim 4.4$ 以及 $\delta \sim 5.8$ 的化学位移归属于半乳糖分子结构中的特征质 子. 此外, 与图 3 对比可知, 在 $\delta 4.5 \sim 4.6$ 处对应于 PEGMA 侧链中一 $\mathrm{CH}_{2} \mathrm{OH}$ 上的质子峰已基本消失, 进一 步验证了半乳糖与聚合物成功键合制得 P(PEGMEMA$c o$-PEGMA-Gal)- $b$-PDMAEMA 共聚物.

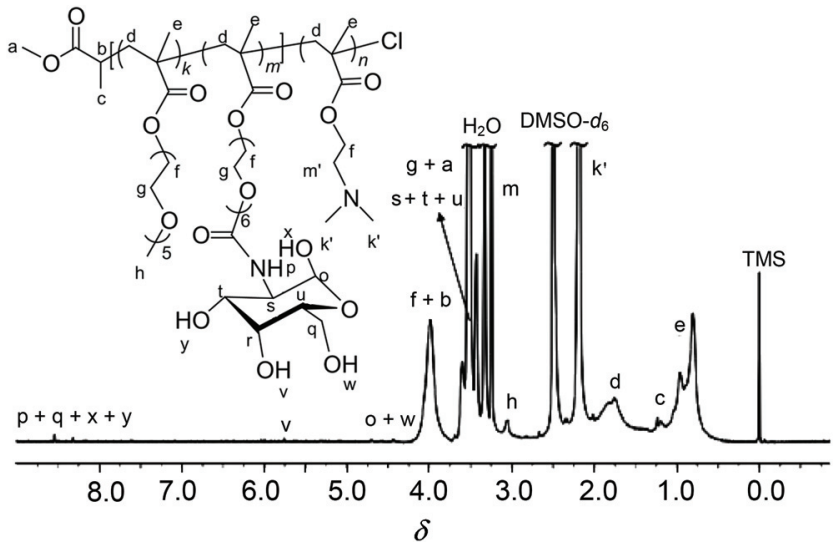

图 $5 \mathrm{P}\left(\mathrm{PEGMEMA}_{16}-c o-\mathrm{PEGMA}_{7-\mathrm{Gal}}\right)-b$-PDMAEMA ${ }_{53}$ 的 ${ }^{1} \mathrm{H}$ NMR 谱 图

Figure $5{ }^{1} \mathrm{H}$ NMR spectrum of $\mathrm{P}\left(\mathrm{PEGMEMA}_{16}-\mathrm{co}-\mathrm{PEGMA}_{7-\mathrm{Gal}}\right)-b-$ PDMAEMA $_{53}$

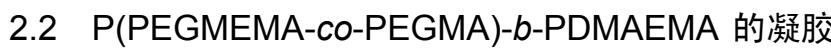 阻滞电泳测试}

凝胶阻滞电泳测试常用于研究基因载体对 DNA 的 压缩能力，即阳离子载体对 DNA 迁移的阻滞能力. PDMAEMA 链段在 $\mathrm{pH} 7.4$ 条件下, 可以部分质子化带 
正电荷, 因而可通过静电相互作用与 DNA 复合. 图 6 为 阳离子型刷形嵌段共聚物 P(PEGMEMA 16 - $c 0$ - PEGMA $\left._{7}\right)$ $b$-PDMAEMA ${ }_{53}$ 对 DNA 的凝胶阻滞电泳结果. 泳道 1 为 裸 DNA, 泳道 2 9 为阳离子嵌段共聚物与 DNA 的复 合物, N/P 比依次为 $0.3,0.6,0.8,1.5,2.5,3.0,8.0$ 和 14 . 从图中可以观察到, 泳道 1 中 DNA 发生完全迁移; 随着 $\mathrm{N} / \mathrm{P}$ 比的增大, DNA 逐渐被阳离子型聚合物固定住, 而 当 N/P 比大于 1.5 时, 即泳道 5 9, DNA 可以完全被固 定住, 说明聚合物中阳离子链段可以完全阻滞 DNA 的 迁移. 上述结果表明, 阳离子型嵌段共聚物能够与 DNA 通过静电相互作用, 压缩 DNA 形成复合物, 作为基因 载体.

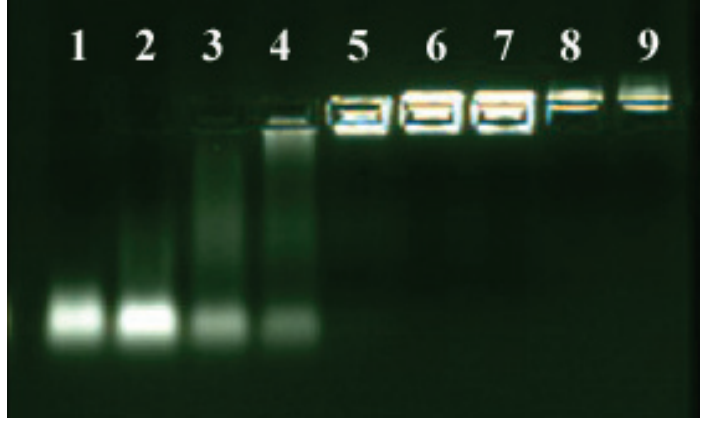

图 6 阳离子型嵌段共聚物 $\mathrm{P}\left(\mathrm{PEGMEMA}{ }_{16}-c o-\mathrm{PEGMA}_{7}\right)-b$ $\mathrm{PDMAEMA}_{53}$ 对 DNA 的凝胶阻滞电泳结果. 泳道 1 为裸 DNA, 泳道 2 9 分别为嵌段共聚物与 DNA 在不同 N/P 比条件下的复合物 $(\mathrm{N} / \mathrm{P}$ 比 分别为 $0.3,0.6,0.8,1.5,2.5,3.0,8.0$ 和 14)

Figure 6 Agarose gel electrophoresis of P(PEGMEMA 16 - $\left.\mathrm{Co}-\mathrm{PEGMA}_{7}\right)$

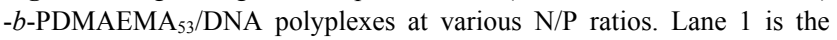
naked DNA control; Lanes 2-9 correspond to N/P ratios of 0.3, 0.6, 0.8, $1.5,2.5,3.0,8.0$ and 14 , respectively

\section{3 复合物的 zeta 电位和粒径测试}

通过 DLS 测试粒子表面的 zeta 电位值, 研究半乳糖 胺修饰的阳离子型刷形嵌段共聚物 P(PEGMEMA ${ }_{16}-c o-$ $\mathrm{PEGMA}_{7-\mathrm{Gal}}$ )- $b$-PDMAEMA 53 与 DNA 的复合物在不同 $\mathrm{N} / \mathrm{P}$ 比条件下表面电荷情况. 图 7 为共聚物/DNA复合物 在不同 N/P 比条件下 zeta 电位的变化. 从图中可以看出, 裸 DNA 的 zeta 电位值约为 $-16.7 \mathrm{mV}$, 随着复合物中聚 阳离子链段的增多, 即 N/P 比值的增大, 复合物表面的 zeta 电位值逐渐由负值变为正值, 并逐渐增加. 当 N/P 比约为 1.0 时, 复合物表面的 zeta 电位值接近中性, 与 凝胶阻滞电泳结果一致. 当 N/P 比为 10.0 的时候, 复合 物表面电位值为 $23.3 \mathrm{mV}$, 从而验证了 $\mathrm{P}\left(\mathrm{PEGMEMA}_{16}-\right.$ $c o-\mathrm{PEGMA}_{7-\mathrm{Gal}}$ )- $b$-PDMAEMA 53 可以与 DNA 发生静电 相互作用, 形成复合物. 同时, 我们也利用 DLS 表征了 嵌段共聚物/DNA 复合物的平均粒径和粒径分布, 结果 如图 8 所示. 从图中可以看出, 复合物的平均粒径为 $106 \mathrm{~nm}$, 粒径分布为 0.150 .

\section{4 细胞毒性测试}

细胞毒性测试是表征生物医用材料生物相容性的 方法之一. 本文采用 MTT 法检测半乳糖胺修饰的阳离

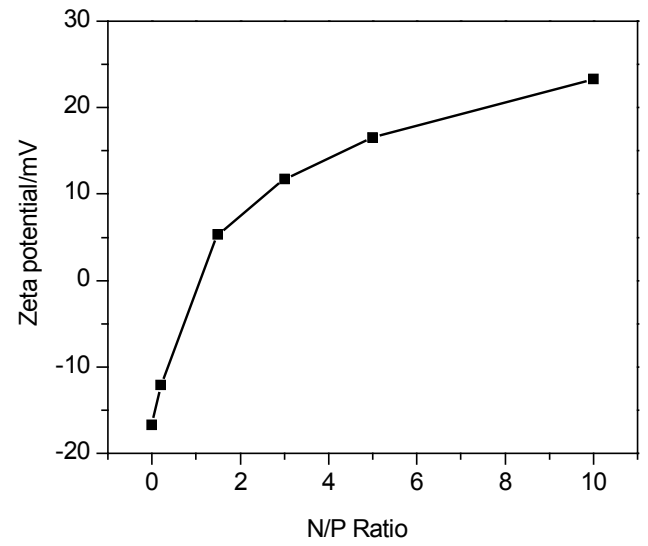

图 $7 \mathrm{P}\left(\mathrm{PEGMEMA}_{16}-c o-\mathrm{PEGMA}_{7-\mathrm{Gal}}\right)-b-\mathrm{PDMAEMA}_{53}$ 与 DNA 复合物 在不同 N/P 比条件下的表面 zeta 电位变化曲线

Figure 7 Zeta potential change of $\mathrm{P}\left(\mathrm{PEGMEMA}_{16}-\mathrm{co}\right.$-PEGMA $\left.{ }_{7-\mathrm{Gal}}\right)-b$ $\mathrm{PDMAEMA}_{53} / \mathrm{DNA}$ polyplexes at various N/P ratios

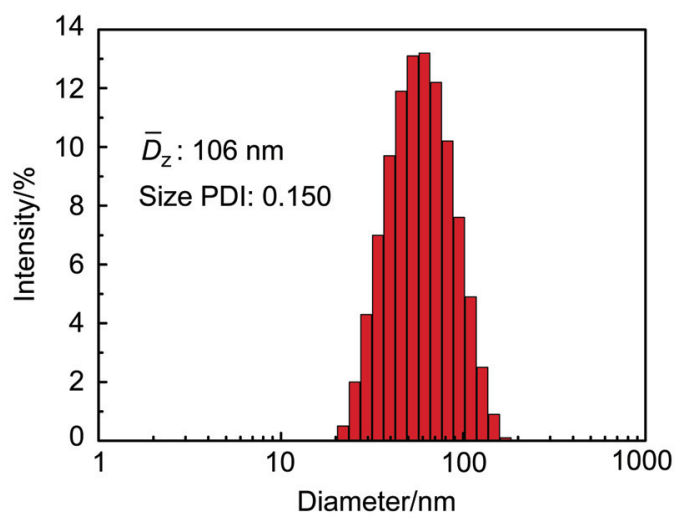

图 $8 \mathrm{P}\left(\mathrm{PEGMEMA}_{16}-c o-\mathrm{PEGMA}_{7-\mathrm{Gal}}\right)-b-\mathrm{PDMAEMA}_{53}$ 与 DNA 复合物 在 N/P 比为 15 时的粒径分布柱状图

Figure 8 The particle size distribution histogram of P(PEGMEMA $16^{-}$ $c o$-PEGMA ${ }_{7-\mathrm{Gal}}$ )- $b$-PDMAEMA ${ }_{53} / \mathrm{DNA}$ polyplexes at a N/P ratio of 15

子型刷形嵌段共聚物 P(PEGMEMA $16-c o-P^{-}$PEMA $\left._{7-G a l}\right)-b$ $\mathrm{PDMAEMA}_{53}$ 对 HepG2 细胞的毒性, 并与商品化的支化 聚乙烯亚胺(PEI, $25 \mathrm{kDa})$ 进行对比. 从图 9 可以看出, 在聚合物浓度较低时 $\left(25 \mu \mathrm{g} \cdot \mathrm{mL}^{-1}\right)$, 对应的细胞存活率 较高; 随着聚合物浓度增加, 由于阳离子型 PDMAEMA 链段增多，毒性逐渐增大，细胞存活率下降. 在较高的 聚合物浓度条件下，浓度为 $200 \mu \mathrm{g} \cdot \mathrm{mL}^{-1}$ 时，与常见的 聚阳离子支化的 PEI 体系对比，我们合成的嵌段共聚物 体系呈现相对稍高的细胞存活率，从而证明该阳离子型 刷形嵌段共聚物比常用的支化 PEI 的毒性低, 具有潜在 的生物应用前景.

\section{5 体外转染测试}

半乳糖分子对哺乳动物肝细胞内一种高效内吞受 体的去唾液酸糖蛋白受体(ASGPR)具有特定的识别作 用, 提高了受体介导的内吞机制和肝靶向运送，从而增 强基因或药物的输送. 我们研究了半乳糖胺修饰的阳离 子型刷形嵌段共聚物 P(PEGMEMA 16 - $\left.c o-\mathrm{PEGMA}_{7-\mathrm{Gal}}\right)-b$ - 


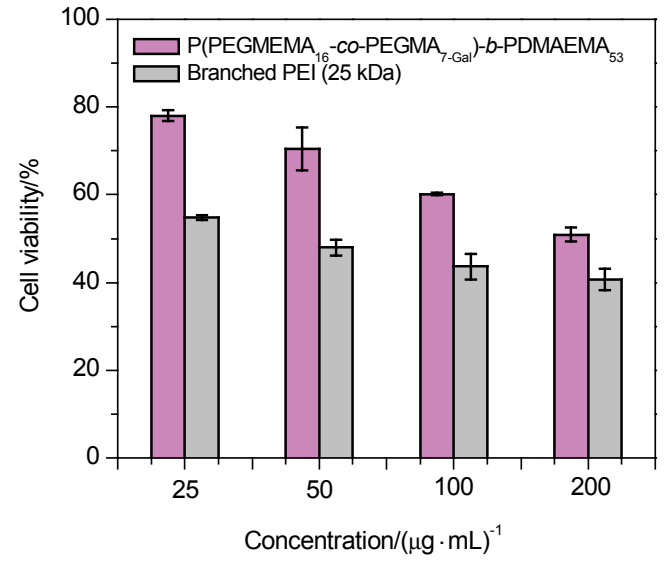

图 $9 \mathrm{P}\left(\mathrm{PEGMEMA}_{16}-c o-\mathrm{PEGMA}_{7-\mathrm{Gal}}\right)-b-\mathrm{PDMAEMA}_{53}$ 与支化 $\mathrm{PEI}(25$ $\mathrm{kDa})$ 对 HepG2 细胞的毒性测试结果

Figure 9 In vitro cytotoxicity study of $\mathrm{P}\left(\mathrm{PEGMEMA}_{16}-\mathrm{co}-\mathrm{PEGMA}_{7-\mathrm{Gal}}\right)$ $-b$-PDMAEMA ${ }_{53}$ and branched PEI $(25 \mathrm{kDa})$ with various concentrations against HepG2 cells by MTT assay

$\mathrm{PDMAEMA}_{53}$ 与 DNA 形成的复合物在 N/P 比为 15 时, 分别对 HeLa 细胞和 HepG2 细胞的转染能力, 结果如图 10 所示, 均可以观察到细胞内绿色苂光蛋白(GFP)标记 的 DNA 的绿色苂光, 说明复合物能够进入细胞, 实现 DNA 的转染. 并且, 从细胞荧光显微镜照片可以看出, 在相同条件下, HepG2 细胞内的绿色荧光比 HeLa 细胞 内的更强, DNA 能够更有效地进入 HepG2 细胞, 说明半 乳糖分子的引入有助于提高肝细胞对载体的内吞作用.
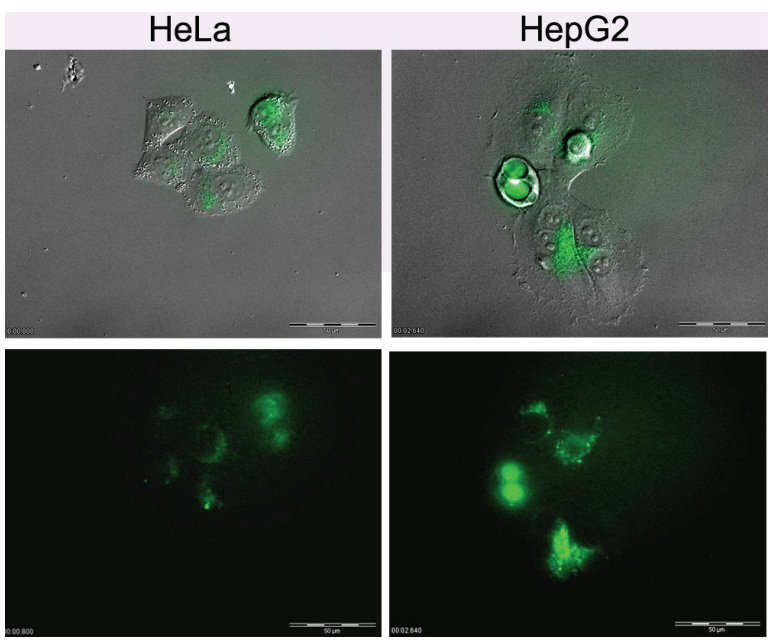

图 $10 \mathrm{P}\left(\mathrm{PEGMEMA}_{16}-c o-\mathrm{PEGMA}_{7-\mathrm{Gal}}\right)-b$-PDMAEMA 53 与 $\mathrm{DNA}$ 的复 合物 $(\mathrm{N} / \mathrm{P}=15)$ 在 HeLa 和 HepG2 细胞中的苂光显微镜照片

Figure 10 The fluorescence microscopy images of HeLa and HepG2 cells treated by the $\mathrm{P}\left(\mathrm{PEGMEMA}_{16}-\mathrm{co}-\mathrm{PEGMA}_{7 \text {-Gal }}\right)-b$-PDMAEMA $\mathrm{P}_{53} /$ DNA polyplexes at a N/P ratios of 15 . Scale bar $=50 \mu \mathrm{m}$

\section{3 结论}

本文结合两步 ATRP 反应和聚合物后修饰法成功制 备了半乳糖胺修饰的阳离子型刷形嵌段共聚物 P(PEGMEMA-co-PEGMA-Gal $)$ - $b$-PDMAEMA. 其中，半
乳糖分子赋予其肝靶向性，刷形 PEG 亲水链段具有抗 蛋白吸附性，而 PDMAEMA 链段在 $\mathrm{pH} 7.4$ 条件下可以 部分质子化带正电荷, 通过静电相互作用与 DNA 复合, 可用作肝靶向基因载体. 凝胶阻滞电泳、zeta 电位和粒 径测试结果表明，该共聚物可以压缩 DNA，形成粒径约 为 $106 \mathrm{~nm}$ 的复合物纳米粒子. 体外毒性测试(MTT 法) 结果表明, 对于 HepG2 细胞, 嵌段共聚物比支化 PEI(25 $\mathrm{kDa}$ )具有更低的细胞毒性. 体外转染测试结果表明, 由 于半乳糖的引入, 聚合物/DNA 复合物对 HepG2 细胞具 有更高的转染能力. 该半乳糖胺修饰的阳离子型刷形嵌 段共聚物具有良好的生物相容性和较强的 DNA 压缩能 力，可望用作靶向基因治疗载体.

\section{4 实验部分}

\section{1 试剂与仪器}

试剂：四氢呋喃(THF，分析纯，国药集团化学试 剂): 用 $\mathrm{KOH}$ 干燥 $3 \mathrm{~d}$, 加入二苯甲酮作为指示剂, 用钠 丝进行无氧回流，直至呈深紫色，使用前蒸出。单体聚 乙二醇单甲醚甲基丙烯酸酯(PEGMEMA, $\bar{M}_{\mathrm{n}} \approx 300$ $\mathrm{g} \cdot \mathrm{mol}^{-1}$ )和聚乙二醇甲基丙烯酸酯 $\left(\mathrm{PEGMA}, \bar{M}_{\mathrm{n}} \approx 360\right.$ $\mathrm{g} \bullet \mathrm{mol}^{-1}$, Sigma-Aldrich): 用 THF 稀释后, 经中性 $\mathrm{Al}_{2} \mathrm{O}_{3}$ 柱子除去阻聚剂, 将流出液旋蒸除去溶剂, 室温真空干 燥后使用。甲基丙烯酸-2-( $N, N$-二甲氨基) 乙 酯 (DMAEMA，工业纯，无锡新宇化工), 在 $\mathrm{CaH}_{2}$ 中干燥 一天后减压蒸馏使用. 以下试剂均直接使用: 无水乙 醇、 $N, N$-二甲基甲酰胺(DMF)、乙醚、二氯甲烷 $\left(\mathrm{CH}_{2} \mathrm{Cl}_{2}\right)$ 、 三乙胺(TEA)、正己烷均为分析纯(国药集团化学试剂); $\alpha$-溴代丙酸甲酯 (MBP, 98\%, Sigma-Aldrich); 氯化亚铜 (CuCl, 99.999\%, Alfa Aser); $N, N, N^{\prime}, N^{\prime}, N^{\prime \prime}$-五甲基二亚乙 基三胺(PMDETA，百灵威科技); Tris-硼酸缓冲液(TBE, 北京索莱宝科技); $N, N^{\prime}$-羰基二咪唑(CDI，苏州昊帆生物 科技); 半乳糖胺盐酸盐( $98 \%$ ，上海聚源生物科技); 小 牛胸腺 DNA(Sigma-Aldrich); DMEM 培养基(Invitrogen); 3-(4,5-二甲基噻唑-2)-2,5-二苯基四氮唑溴盐 (MTT, 98\%, Sigma-Aldrich).

仪器: 核磁共振波谱仪(NMR, INOVA-400, Varian); 红外光谱仪(FT-IR, Nicolet 6700), 溴化钾压片制样; 凝 胶渗透色谱仪(GPC, Waters 1515, Waters), 测定时以聚 苯乙烯为标样, 流动相为 $\mathrm{THF}$, 流动速率为 $1.0 \mathrm{~mL}$ $\mathrm{min}^{-1}$, 测试温度为 $30{ }^{\circ} \mathrm{C}$; 动态激光光散射仪(Zetasizer Nano-ZS, Malvern), 测试温度为 $25{ }^{\circ} \mathrm{C}$, 利用 Zetasizer 软件进行数据处理; 酶标仪(PowerWave XS, Bio-Tek); 活细胞工作站系统(CELL'R, Olympus).

\section{2 聚合物的合成}

\subsubsection{P(PEGMEMA-co-PEGMA)的合成}

依次取 PEGMEMA (3.15 g, $10.5 \mathrm{~mol})$, PEGMA (1.62 g, $4.5 \mathrm{mmol}), 10 \mathrm{~mL}$ 无水乙醇、引发剂 MBP (56.8 
$\mu \mathrm{L}, 0.5 \mathrm{mmol})$, 配体 PMDETA $(208 \mu \mathrm{L}, 1 \mathrm{mmol}) 、$ 催化 剂 $\mathrm{CuCl}(0.0495 \mathrm{~g}, 0.5 \mathrm{mmol})$ 于干燥的支管瓶中, 充放高 纯氮气三次以除去瓶中的氧气，最后充入高纯氮气，将 密封的反应瓶置于 $30{ }^{\circ} \mathrm{C}$ 油浴中搅拌 $5 \mathrm{~h}$. 反应结束后, 加入 $50 \mathrm{~mL}$ 无水乙醇稀释反应物, 使之通过装有中性 $\mathrm{Al}_{2} \mathrm{O}_{3}$ 的柱子以除去铜盐, 旋转蒸发使溶液浓缩, 加入 $20 \mathrm{~mL}$ 超纯水稀释, 用超纯水透析(MWCO 3500) $24 \mathrm{~h}$ 后, 经冷冻干燥得到浅黄色粘状物质(产率 86.7\%).

4.2.2 P(PEGMEMA-co-PEGMA)- $b$-PDMAEMA 的合成 以无规共聚物 P(PEGMEMA-co-PEGMA)作为大分 子引发剂, 引发单体 DMAEMA 聚合. 依次称取 P(PEGMEMA-co-PEGMA) (1.87 g, $0.25 \mathrm{mmol}), 15 \mathrm{~mL}$ 无水乙醇, PMDETA (104 $\mu \mathrm{L}, 0.5 \mathrm{mmol}), \mathrm{CuCl}(0.0258 \mathrm{~g}$, $0.25 \mathrm{mmol}$ )加入干燥的 $50 \mathrm{~mL}$ 支管瓶中, 充放高纯氮气 三次, 最终充入高纯氮气, 并用针筒注入 DMAMEA (2.36 g, $15 \mathrm{mmol})$, 将密封的反应瓶置于 $30{ }^{\circ} \mathrm{C}$ 油浴中摚 拌 $5 \mathrm{~h}$. 反应结束后, 加入 $50 \mathrm{~mL}$ 无水乙醇稀释反应产 物, 使之通过装有中性 $\mathrm{Al}_{2} \mathrm{O}_{3}$ 的柱子以除去铜盐, 旋转 蒸发使溶液浓缩. 用冰正己烷沉淀后, 于 $25{ }^{\circ} \mathrm{C}$ 真空烘 箱中干燥至恒重, 得到浅黄色透明粘状固体 (产率 $58.8 \%$ ).

\subsubsection{P(PEGMEMA-co-PEGMA-Gal)- $b$-PDMAEMA 的} 合成

首先, 依次称取 P(PEGMEMA-co-PEGMA)- $b$ PDMAEMA (2.155 g, $0.1 \mathrm{mmol})$, CDI (0.227 g, 0.5 $\mathrm{mmol}$ )加入干燥的 $50 \mathrm{~mL}$ 支管瓶中, 充放高纯氮 3 次, 最 终充入高纯氮气; 并用针筒注入 $25 \mathrm{~mL}$ 无水 $\mathrm{THF}$, 充分 搅拌至溶解, 在 $25{ }^{\circ} \mathrm{C}$ 下反应 $12 \mathrm{~h}$. 反应结束后, 旋蒸除 去 THF, 在正己烷与无水乙醚体积比为 $8: 2$ 的混合溶 剂中沉淀得到白色粘状物质, 于 $25{ }^{\circ} \mathrm{C}$ 真空烘箱中干燥 至恒重, 得到浅黄色透明粘状固体 P(PEGMEMA-co-

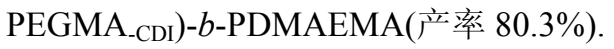

随后, 称取半乳糖胺盐酸盐 $(0.05 \mathrm{~g}, 0.231 \mathrm{mmol})$ 和 P(PEGMEMA-co-PEGMA_CDI)- $b$-PDMAEMA $\quad(0.2 \mathrm{~g}$, $0.022 \mathrm{mmol}$ ) 溶解于 $10 \mathrm{~mL}$ DMF 并加入干燥的 $50 \mathrm{~mL}$ 支 管瓶中. 向恒压滴液漏斗中加入 $50 \mu \mathrm{L}$ TEA 和 $10 \mathrm{~mL}$ $\mathrm{DMF}$, 在 $30 \mathrm{~min}$ 内逐渐滴加到支管瓶中, 并在 $25{ }^{\circ} \mathrm{C}$ 下 反应 $24 \mathrm{~h}$. 反应完毕后, 用超纯水透析(MWCO 1000)12 $\mathrm{h}$, 经冷冻干燥得到乳白色固体(产率 $72 \%$ ).

\section{3 凝胶阻滞电泳测试}

将聚合物与 DNA 分别溶解在 $0.5 \times \mathrm{TBE}$ 缓冲液中, 配成浓度均为 $1 \mathrm{mg} \cdot \mathrm{mL}^{-1}$ 的聚合物和 DNA 溶液. 根据 不同的 N/P 比, 将 DNA 溶液与聚合物溶液混合, 漩浴震 荡使其混合均匀. 通过静电相互作用形成聚合物与 DNA 的复合物溶液. 将上述复合物溶液 $(8 \mu \mathrm{L})$ 与 $2 \mu \mathrm{L}$ 的上样缓冲溶液 $(85 \%$ 丙三醇和 $15 \%$ 溴酚蓝)混合, 点样 到含有 $0.5 \mu \mathrm{g} \cdot \mathrm{mL}^{-1}$ 溴乙锭 $0.8 \%$ 的琼脂糖凝胶中. 电泳 电压为 $90 \mathrm{~V}$, 时间为 $30 \mathrm{~min}$, 缓冲溶液为 $0.5 \times \mathrm{TBE}$. 利
用紫外光仪(M-15E, UVP)观察凝胶中 DNA 的迁移.

\section{4 细胞毒性测试(MTT 法)}

利用 MTT 法检测载体的细胞毒性. 将人肝癌细胞 (HepG2)置于由 $10 \%$ 热灭活性胎牛血清、1\%青霉素和链 霉素组成的 DMEM 培养基中, 在 $5 \%$ 的 $\mathrm{CO}_{2}$ 气体和 37 ${ }^{\circ} \mathrm{C}$ 条件下培养. 首先, 在 96 孔板中将细胞以每孔 50000 个细胞的密度排布于 $100 \mu \mathrm{L}$ 的含有 $10 \%$ 血清的 DMEM 培养基中培养 $24 \mathrm{~h}$. 将不同浓度的聚合物溶液分别加入 不同的孔内与细胞一起培养 $24 \mathrm{~h}$. 接着, 在每个孔中加 入 $20 \mu \mathrm{L}$ 的 MTT 溶液 $\left(5 \mathrm{mg} \cdot \mathrm{mL}^{-1}\right)$, 进一步培养 $4 \mathrm{~h}$. 最 后，吸走培养基并加入 $150 \mu \mathrm{L}$ DMSO，通过酶标仪测试 每一个孔在 $570 \mathrm{~nm}$ 的吸光度(OD). 细胞的相对毒性即 为 $[\mathrm{OD}]_{\text {test }} /[\mathrm{OD}]_{\text {control }} \times 100 \%$, 其中, $[\mathrm{OD}]_{\text {test }}$ 为聚合物溶 液存在下的吸收值; $[\mathrm{OD}]_{\mathrm{control}}$ 为没有加聚合物溶液的参 照组的吸收值.

\section{5 体外转染测试}

分别将 HeLa 和 HepG2 细胞置于由 $10 \%$ 热灭活性胎 牛血清、 $1 \%$ 青霉素和链霉素组成的 DMEM 培养基中, 在 $5 \%$ 的 $\mathrm{CO}_{2}$ 气体和 $37{ }^{\circ} \mathrm{C}$ 条件下培养. 细胞接种在仪器 配套专用的培养血 $(\Phi 35 \mathrm{~mm})$ 中贴壁生长, 培养 $24 \mathrm{~h}$. 按不同的混合比例来转染细胞. 在培养血中加入合适体 积的 DMEM 培养基，一定量不同 N/P 比的复合物混合 后震荡, 每孔加入 $100 \mu \mathrm{L}$ 上述混合液, 轻轻地摇晃培养 III, 在 $37{ }^{\circ} \mathrm{C}$ 和 $5 \% \mathrm{CO}_{2}$ 条件下培养 $6 \mathrm{~h}$, 吸走培养血中 的培养基，再加入 $500 \mu \mathrm{L}$ 新鲜的培养基，将细胞放回培 养箱中下继续培养 $30 \mathrm{~h}$. 通过活细胞工作站系统观察细 胞中 DNA 的绿色荧光表达情况.

\section{References}

[1] O’Neal, W. K.; Zhou, H. S.; Morral, N.; Langston, C.; Parks, R. J.; Graham, F. L.; Kochanek, S.; Beaudet, A. L. Mol. Med. 2000, 6, 179.

[2] Mintzer, M. A.; Simanek, E. E. Chem. Rev. 2009, 109, 259

[3] Xu, C. H.; Sui, M. H.; Tang, J. B.; Shen, Y. Q. Chin. J. Polym. Sci. 2011, 29, 274.

[4] Yue, Y. N.; Wu, C. Biomater. Sci. 2013, 1, 152.

[5] Gosselin, M. A.; Guo, W. J.; Lee, R. J. Bioconjugate Chem. 2001, $12,989$.

[6] Wang, J.; Mao, H. Q.; Leong, K. W. J. Am. Chem. Soc. 2001, 123, 9480.

[7] Wang, D. A.; Narang, A. S.; Kotb, M.; Gaber, A. O.; Miller, D. D.; Kim, S. W.; Mahato, R. I. Biomacromolecules 2002, 3, 1197.

[8] Forrest, M. L.; Koerber, J. T.; Pack, D. W. Bioconjugate Chem. 2003, 14, 934 .

[9] Zhang, W. D.; Cheng, Q.; Guo, S. T.; Lin, D. S.; Huang, P. S.; Liu, J.; Wei, T.; Deng, L. D.; Liang, Z. C.; Liang, X. J.; Dong, A. J. Biomaterials 2013, 34, 6495.

[10] Wang, Y.; Wang, L. S.; Goh, S. H.; Yang, Y. Y. Biomacromolecules 2007, 8, 1028.

[11] Zhao, X. B.; Lee, R. J. Adv. Drug Delivery Rev. 2004, 56, 1193.

[12] Chiu, S. J.; Ueno, N. T.; Lee, R. J. J. Controlled Release 2004, 97, 357.

[13] Pan, X. G.; Wu, G.; Yang, W. L.; Barth, R. F.; Tjarks, W.; Lee, R. J. Bioconjugate Chem. 2007, 18, 101.

[14] Shen, Y.; Hu, G. X.; Zhang, H. X.; Qi, L. L.; Luo, C. C. Acta Chim. Sinica 2013, 71, 323. (沈银, 胡桂香, 张华星, 齐莉莉, 骆成才, 化学学报, 2013, 71, 323.)

[15] Cui, L.; Li, Y.; Hou, X. D.; Gong, W. J.; Xu, Y. H.; Cao, A. M. Acta 
Chim. Sinica 2007, 65, 2181. (崔亮, 李洋, 侯小东, 宫文娟, 徐宇 虹, 曹阿民, 化学学报, 2007, 65, 2181.)

[16] Sato, A.; Choi, S. W.; Hirai, M.; Yamayoshi, A.; Moriyama, R.; Yamano, T.; Takagi, M.; Kano, A.; Shimamoto, A.; Maruyama, A. J. Controlled Release 2007, 122, 209.

[17] Tria, M. C. R.; Grande, C. D. T.; Ponnapati, R. R.; Advincula, R. C. Biomacromolecules 2010, 11, 3422.

[18] Zhou, Z. L.; Yu, P. P.; Geller, H. M.; Ober, C. K. Biomacromolecules 2013, 14, 529.

[19] Liang, H. F.; Chen, C. T.; Chen, S. C.; Kulkarni, A. R.; Chiu, Y. L.; Chen, M. C.; Sung, H. W. Biomaterials 2006, 27, 2051.

[20] Jain, K.; Kesharwani, P.; Gupta, U.; Jain, N. K. Biomaterials 2012,
33,4166 .

[21] Nicolas, J.; Mura, S.; Brambilla, D.; Mackiewicz, N.; Couvreur, P. Chem. Soc. Rev. 2013, 42, 1147.

[22] Wu, J.; Sun, T. M.; Yang, X. Z.; Zhu, J.; Du, X. J.; Yao, Y. D. Xiong, M. H.; Wang, H. X.; Wang, Y. C.; Wang, J. Biomater. Sci. 2013, $1,1143$.

[23] Zhai, S. J.; Wang, B. D.; Feng, C.; Li, Y. J.; Yang, D.; Hu, J. H.; Lu, G. L.; Huang, X. Y. J. Polym. Sci. Part A: Polym. Chem. 2010, 48, 647.

[24] Zhai, S. J.; Song, X. M.; Feng, C.; Jiang, X. Y.; Li, Y. J.; Lu, G. L.; Huang, X. Y. Polym. Chem. 2013, 4, 4134. 